\title{
Le Roman du temps perdu. Le mythe de Proust et la recherche de Barthes
}

\section{Claudia Amigo Pino}

\section{(2) OpenEdition}

1 Journals

Édition électronique

URL : http://journals.openedition.org/recherchestravaux/424

DOI : 10.4000/recherchestravaux.424

ISSN : 1969-6434

Éditeur

UGA Éditions/Université Grenoble Alpes

Édition imprimée

Date de publication : 20 décembre 2010

Pagination : 45-56

ISBN : 978-2-84310-187-8

ISSN : 0151-1874

Référence électronique

Claudia Amigo Pino, «Le Roman du temps perdu. Le mythe de Proust et la recherche de Barthes », Recherches \& Travaux [En ligne], 77 | 2010, mis en ligne le 20 août 2012, consulté le 08 septembre 2020. URL : http://journals.openedition.org/recherchestravaux/424 ; DOI : https://doi.org/10.4000/ recherchestravaux.424 
Claudia Amigo Pino

Université de São Paulo

\section{Le Roman du temps perdu Le mythe de Proust et la recherche de Barthes}

\section{Introduction : le mythe de la vie nouvelle}

Le 25 février 1980, vers I5 h 40, Roland Barthes traversait la rue des Écoles. Selon les témoins, il avait apparemment regardé des deux côtés avant de s'engager. Qu'il ait vu ou non la camionnette qui l'a renversé, cette incertitude a contribué à nourrir le mythe de l'œuvre qu'il écrivait alors, le roman Vita Nova. Dans le sillage de Pascal et Mallarmé, Barthes travaillait, en effet, depuis 1978 à une œuvre totale, absolue, qui inaugurerait une nouvelle étape de sa vie, voire une vie nouvelle (c'est le sens de Vita Nova ${ }^{\mathrm{r}}$ ). Dans le cadre de la conférence «Longtemps je me suis couché de bonne heure», l'auteur aborde clairement ce projet :

Est-ce que tout cela veut dire que je vais écrire un roman? Je n'en sais rien. Je ne sais s'il sera possible d'appeler encore "roman" l'œuvre que je désire et dont j'attends qu'elle rompe avec la nature uniformément intellectuelle de mes écrits passés (même si bien des éléments romanesques en altèrent la rigueur). Ce Roman utopique, il m'importe de faire comme $s i$ je devais l'écrire².

À l'instar du projet pascalien cherchant à démontrer l'existence de Dieu, les prétentions de cette œuvre à venir ne sont guère modestes. Selon ce que Barthes lui-même a suggéré, elle devait accomplir les trois missions que $\mathrm{La}$

I. Dans les Euvres complètes de Barthes, éditées par É. Marty, «Vita Nova» est le titre d'un ensemble de huit feuillets manuscrits trouvés dans une chemise et dont les fac-similés, assortis d'une transcription, sont reproduits à la fin du dernier volume. Voir R. Barthes, Euvres complètes, É. Marty (éd.), Seuil, t. V, 2002, p. 994-IOOI et p. I007-IOI8. Les références donneront désormais $O C$, et le numéro du tome auquel se reporter.

2. R. Barthes, «Longtemps je me suis couché de bonne heure», $O C$, t. V, p. 470. 
Préparation du roman définit au début de sa démarche : I) faire vivre ou revivre des personnes aimées ; 2) dire l'amour et 3) entendre l'amour de l'autre’.

Personne n'a jamais lu ce roman, et certains suggèrent même que l'impossibilité de l'écrire a tari chez l'auteur le désir de vivret. Barthes est-il vraiment entré dans l'écriture de cette œuvre ou a-t-il seulement fait semblant? Selon le Barthesprofesseur de La Préparation, la réponse serait : «non, je ne l'ai pas écrite»:

En effet, quelle serait la conclusion de ce cours? - L'œuvre elle-même. Dans un bon scénario, la fin matérielle du Cours aurait dû coïncider avec la publication réelle de l'œuvre dont nous avons suivi le cheminement à même son projet, sa volonté.

Hélas, en ce qui me concerne, il n'en est pas question : je ne puis sortir aucune Euvre de mon chapeau, et de toute évidence sûrement pas ce Roman dont j'ai voulu analyser la préparations.

Éric Marty, éditeur des Euvres complètes, rappelle que Barthes a quand même écrit quelque chose - à première vue, très peu de chose : «De ce projet, nous n'avons que huit feuillets, huit esquisses, huit plans que nous reproduisons à la fin du volume en fac-similléc." Si, à partir de ces feuillets, certains critiques ont essayé de résoudre la question de cette ouvre en projet, invariablement, la réponse a toujours été négative. Je ne donnerai ici que deux exemples. D'abord, Diana Knight, qui soutient que l'œuvre de Barthes ne pouvait être écrite, pour la simple raison que les plans finissent sur un éloge $\mathrm{du}$ «ne rien faire». On rencontre cet éloge, par exemple, à la fin du deuxième feuillet :

VII L'Oisivité pure : le «rien faire philosophique». (Le neutre, le Tao / le Tas.

- Les Amis (Fantasme de ne s'occuper que d'eux)

- Le retour aux places antérieures. Continuer. Pas de V[ita] N[ova] $]^{7}$.

La réflexion de Diana Knight, cependant, loin de livrer des certitudes, pose plutôt le problème suivant : comment peut-on dire qu'une œuvre n'est pas faite, si faire l'œuvre - selon ses plans - signifie «ne rien faire»?

3. "Cette forme, je ne puis la soumettre par avance aux règles structurales du Roman. Je puis seulement lui demander de remplir à mes propres yeux trois missions : La première serait de me permettre de dire ceux que j'aime [...], et non pas de leur dire que je les aime [...]. La seconde mission que je confierais à ce Roman (fantasmé et probablement impossible), ce serait de permettre la représentation d'un ordre affectif, pleinement, mais indirectement. [...] Enfin et peut-être surtout, le Roman, puisque son écriture est médiate, le Roman, donc, ne fait pas pression sur l'autre; son instance est la vérité des affects, non des idées [...].» (Ibid., p. 47I.)

4. C'est le cas d'A. Compagnon, qui dans l'article "Le roman de Roland Barthes» (Critique, $\mathrm{n}^{\circ} 678,2003$, p. 790), suggère que Barthes avait perdu l'inspiration et la vigueur pour inventer une œuvre et une vie nouvelle.

5. R. Barthes, La Préparation du roman, N. Léger (éd.), Seuil/IMEC, 2003, p. 377.

6. É. Marty, «Présentation", dans $O C, \mathrm{t} . \mathrm{V}, \mathrm{p} .22$.

7. R. Barthes, "Vita Nova», art. cité, p. IoIo. 
This apparently contradictory statement which cannot, in this context, be read as some modest disclaimer, may well encapsulate the bizarre status of Barthes's project. In what sense can a fantasy fail unless failure is in some sense part of the fantasy?

Pour Jean-Pierre Martin, en revanche, l'échec du projet est dû à sa trop large intertextualités. À partir de la lecture de La Préparation du roman, il affirme que Barthes se serait perdu dans les exemples travaillés dans les cours, exemples d'écriture romanesques ou de changements de vie. "Étrangement, dans cet inventaire raisonné, la vita nova personnelle s'est progressivement éloignée ${ }^{\mathrm{Io}}$." Ce que Jean-Pierre Martin n’a pas pris en compte, c'est que l'écriture du roman ne se résume pas au cours proféré par Barthes au Collège de France : outre les huit esquisses reprises dans les Euvres complètes, d'autres documents peuvent être intégrés au dossier de cette œuvre en devenir. Éric Marty lui-même indique l'existence de ce matériel :

Ce possible [l'œuvre Vita Nova] est en partie accompli lorsque Barthes écrit ces lignes, par l'œuvre déjà écrite; ce possible se nourrit également des tentatives, auxquelles il se prête, d'une autre écriture (le «journal», les fragments, les notations, les épiphanies...), il se nourrit de ce projet même, Vita Nova, mais également, par contre-coup, du cours qu'il donne au Collège de France sur «La Préparation du roman» ou de telle conférence, de tel article, des amis"

Certains de ces documents sont déjà publiés (c'est le cas des journaux, par exemple : Journal de deuil, Incidents, «Soirées de Paris», "Délibération $»^{12} .$. ), mais une grande part reste inédite et se trouve dans le grand fichier Roland Barthes, déposé à l'IMEC ${ }^{13}$. Nous voudrions, dans le cadre de cet article, prendre en compte ces documents, dans le dessein de montrer que Barthes a laissé des possibilités d'écriture. Notre propos sera de présenter une de ces possibilités : la réécriture de $\hat{A}$ la recherche $d u$ temps perdu à la lumière des Mythologies.

8. "Cette déclaration, apparemment contradictoire, et ne pouvant, dans ce contexte, être lue comme un simple renoncement, pourrait résumer la situation étrange du projet barthésien. Dans quel sens un fantasme peut échouer si l'échec est inclus dans ce fantasme?», (D. Knight, «Idle thoughts: Barthes' Vita Nova», dans Nottingham French Studies, printemps 1997, p. 91.)

9. On peut noter que cet argument est également utilisé par L. Nunez, dans son article sur le dossier "Barthes refait signe» (Magazine littéraire, $\mathrm{n}^{\circ}$ 482, janvier 2009).

IO. J.-P. Martin, "Barthes et la "Vita Nova" ", dans Poétique, n 156, novembre 2008.

II. É. Marty, «Présentation", art. cité, p. 2I-22.

I2. Journal de deuil est paru au Seuil, en 2009. "Incidents» et "Soirées de Paris» ont été publiés par Fr. Wahl dès 1987 dans le recueil Incidents, chez le même éditeur. «Délibération» fut d'abord publié dans Tel Quel en 1979. Ces trois textes sont repris dans le tome V des Euvres complètes.

I3. Je remercie vivement M. Michel Salzedo, qui m'a permis la consultation des documents du Fichier Roland Barthes, alors conservé à l'Institut de mémoire de l'édition contemporaine (IMEC). Les archives de Barthes ont été transférées à la Bibliothèque nationale en septembre 2010. 


\section{Mythologies du roman}

\section{Le mythe de Proust}

Il est tout à fait possible d'envisager Vita Nova comme un mythe, le mythe du roman assassin, qui serait le vrai coupable de la mort de son auteur. Ce paradoxe rappelle les propos de Barthes, selon lesquels l'écriture est anéantissement, silence, et mort de tout auteur. Il rappelle également les préoccupations de Barthes concernant, notamment, la voix de la mort dans le récit, qu'il s'agisse des contes de Poe ou d'À la Recherche du temps perdu.

Ce travail étant en partie déjà menér ${ }^{14}$, je propose ici d'interroger un autre mythe, celui de La Recherche comme ouvre désirable, ouvre que l'auteur voulait récrire. Comme le Pierre Ménard de Borgès, qui voulait écrire $L e$ Quichotte avec les mêmes mots que Cervantès et produire cependant une œuvre originale, Barthes affirme son désir d'écrire sur le même sujet que la Recherche (le désir d'écrire) et d'utiliser la même forme (qui vacillerait entre le roman et l'essai $\left.{ }^{\mathrm{Is}}\right)$.

C'est le propos de la plupart des manuscrits que j'ai pu consulter à l'IMEC, et c'est aussi la direction que Barthes prenait au moment de son accident, en février I980. Il s'était, en effet, rendu au Collège de France pour régler certains aspects techniques concernant le séminaire "Proust et la photographie», qu'il devait commencer quelques jours après, et dans lequel il souhaitait étudier la manière proustienne de transformer des personnes «réelles» en personnages.

\section{Les mythes de Barthes}

Avant de se demander pourquoi l'œuvre de Proust constitue un mythe pour Barthes, il faut revenir sur le concept de «mythe» développé dans Mythologies, en 1957. Barthes définit alors le mythe comme le contraire de l'interprétation littéraire : tandis que l'interprétation littéraire essaie d'établir un chemin du récit à ce que nous appelons «la réalité», la pensée du mythe selon Barthes essaie d'établir un chemin de "la réalité» au récit. Autrement dit, il s'agit, pour le mythe, de transformer la réalité en récit.

D'après les Mythologies, ce que nous appelons «la réalité» pourrait être défini comme autant d'usages sociaux : "pratiquées nationalement, les normes bourgeoises sont vécues comme les lois évidentes d'un ordre naturel : plus la classe bourgeoise propage ses représentations, plus elles se naturalisent $t^{16}{ }$. Barthes donne l'exemple du grand mariage bourgeois, dont la fonction

I4. Je renvoie à l'article déjà cité de D. Knight, "Idle thoughts: Barthes' Vita Nova ».

I5. R. Barthes, "Longtemps je me suis couché de bonne heure", art. cité, p. 459-465.

I6. R. Barthes, Mythologies, $O C$, t. I, p. 852. 
initiale (présenter et consommer les richesses) n'a aucun sens pour les couples issus des classes moyennes et, à plus forte raison, pour les couples les plus pauvres :

En répandant ses représentations à travers tout un catalogue d'images collectives à usage petit-bourgeois, la bourgeoisie consacre l'indifférenciation illusoire des classes sociales : c'est à partir du moment où une dactylo à vingt-cinq mille francs par mois se reconnaît dans le grand mariage bourgeois que l'ex-nomination bourgeoise atteint son plein effet ${ }^{17}$.

En devenant "naturel», "désirable» par les classes moyennes, le mariage perd son sens politique. Et nous voici face à la définition du mythe selon Barthes : «une parole dépolitisée ${ }^{18}$ ». Le rôle du mythologue sera alors de repolitiser cette parole, de la contextualiser, pour lui rendre une fonction, une fin, une histoire. En un mot, pour la réintégrer dans un récit.

Lors de la seconde édition des Mythologies, en 1970, Barthes ne se reconnaît plus dans ce genre de critique : «[...] la critique idéologique, en même temps que l'exigence en ressurgissait brutalement (mai 1968), s'est subtilisée ou du moins demande à l'être ${ }^{19}$ ». Mais, même éloigné de son interprétation marxiste du début, il n'abandonne pas pour autant son activité de mythologue : il est, ainsi, particulièrement intéressant de s'arrêter sur la nouvelle fonction du mythe dans Fragments d'un discours amoureux. Dans ce livre, l'objet ne sera plus le mariage bourgeois, mais ce qui le précède, l'amour, ou le discours amoureux. Or, l'amour, qui, pour la société bourgeoise, est présenté comme un sentiment naturel, devient, selon la proposition de Barthes au début du livre, "une place de parole» : "la place de quelqu'un qui parle en lui-même, amoureusement, face à l'autre (l'objet aimé), qui ne parle pas ${ }^{20}$ ». L'amour est ainsi le contraire de ce qu'affirme la société bourgeoise : ce n'est qu'un cri de solitude.

Cependant, tout en dénonçant le caractère mythique de l'amour, Barthes décide de plonger dans ce mythe, de répéter ce discours, de récrire le Werther, qui est l'un des textes-tuteurs des Fragments. Cela, afin de produire, son propre discours amoureux, ou au moins des fragments de ce discours. Dans ce geste, Barthes abandonne son passé de théoricien, d'intellectuel-phare pour toute une génération, comme Éric Marty l'a développé dans la dernière partie de son livre Roland Barthes, le métier d'écrire:

Ce qui est fondamentalement irrecevable pour la theoria, c'est le langage faible de l'amoureux, ce que la vérité n'y est plus la sienne, celle de son langage, mais

I7. Ibid., p. $852-853$.

I8. Ibid., p. 854 .

19. R. Barthes, "Mythologies (Présentation de la seconde édition)», OC, t. I, p. 673.

20. R. Barthes, Fragments d'un récit amoureux, OC, t. V, p. 29. 
celle du langage de l'amoureux qui n'est ni sujet de la science, ni le Maître, ni l'universitaire, mais qui, par la douceur et la posture, ne pourra jamais produire des énoncés intégrables à l'immense liste des énoncés modernes ${ }^{21}$.

\section{À la recherche de Proust}

On peut retrouver cette même contradiction dans les manuscrits de Vita Nova : tout en essayant de récrire l'œuvre de Proust, Barthes procède à une critique du mythe de La Recherche. Pour rendre compte de ce double mouvement, je voudrais commencer par le désir de mythe en me référant à la conférence "Longtemps je me suis couché de bonne heure...", à la transcription du cours et à quelques entrées du grand fichier Barthes. Il n'est, bien sûr, pas possible de présenter ces documents sans dialoguer avec l'ensemble des écrits de Barthes.

\section{Du côté du mythe : réecrire Proust}

Dès le début, $A$ la Recherche du Temps Perdu s'impose à Barthes comme modèle :

La Recherche est le récit d'un désir d'écrire : je ne m’identifie pas avec à l'auteur prestigieux d'une œuvre monumentale mais à l'ouvrier, tantôt tourmenté, tantôt exalté, de toute manière modeste, qui a voulu entreprendre une tâche à laquelle, dès l'origine de son projet, il a conféré un caractère absolu ${ }^{22}$.

Le fichier de travail dévoile ainsi une entreprise obsessionnelle, presque folle. J'ai pu consulter au moins trois cents fiches, dans lesquelles Barthes essaie de noter, par exemple, tous les lieux fréquentés par les personnages de Proust (et leurs correspondants «réels»), avant d'en dresser le plan et de déterminer la distance qui les sépare. Si le fichier n'est pas, pour l'instant, consultable dans son ensemble, il est à peu près certain qu'existent d'autres documents concernant ce projet. Il est dès lors possible d'avancer quelques hypothèses relatives au contenu de ce qui n'est pas encore disponible. Barthes aurait élaboré deux types de fiches : celles où il explore le «je» (la façon d'utiliser le récit proustien) et celles où il explore le «moi» (ses amis, les personnes qu'il rencontrait dans les cafés, les gigolos, ses affaires).

Concernant le «je», voici quelques directions explorées par les documents. D'abord, l'idée d'un roman à personnages : au colloque de Cerisy de 1977 , Barthes avait déjà avoué que son désir d'écrire un roman était aussi un désir de "peindre ceux que j'aime», et l'on voit clairement dans les fiches

2I. É. Marty, Roland Barthes, le métier d'écrire, Seuil, 2006, p. 205.

22. «Longtemps je me suis couché de bonne heure», R. Barthes, art. cité, p. 459. 
qu'il voulait transformer ses amis, ou les personnes qu'il fréquentait, en personnages. S'inspirant de Proust, ce qu'il voulait transposer, c'était le principe de composition des personnages, ce qu'il appelle le "syntagme proustien", c'est-à-dire l'inversion des "essences» au cours du récit :

- Syntagme proustien :

- des tableaux, des scènes (langagières), des plages : phase de la manifestation d'essence du personnage : tout est fait pour le douer d'une essence (immobile, éternelle)

- puis bcp plus loin, le personnage apparait renversé, il est doué d'une autre essence $^{23}$.

Une écriture en «marcottes», ensuite : selon Barthes, Proust travaillait à partir de fragments qu'il agençait par la suite, de «marcottes» qui s'attachaient progressivement aux autres parties pendant le processus d'écriture. Barthes voulait procéder de façon identique avec ses propres fragments. Je cite un exemple qu'il reprend plusieurs fois : «il [Proust] composait des "morceaux" - ce n'était pas linéaire - qu'il mettait ensuite en ordre - avec augmentation comme une mayonnaise $e^{24} »$.

Une vie nouvelle grâce à la lecture, enfin : ses recherches le conduisent en effet à croire que Proust avait écrit à partir d'un autre texte, le Contre SainteBeuve. Toujours fidèle à son modèle, Barthes était persuadé que l'écriture de son propre roman se déclencherait aussi à partir de la lecture. Et ce premier texte qui le mènerait vers le vrai roman ne serait-il pas "Vita Nova», envisagé comme un véritable relais d'écriture? C'est du moins l'hypothèse que l'on peut formuler à la lecture du passage suivant :

une lecture de la décision d'écrire une lecture : Ste Beuve.

face agissante d'un texte-lu de ce qui Un texte qui est xx plus (ou autre chose) qu'un texte excitant, incitateur (xxx car alors simple psychologie esthetique

[Verso]

de l'écrire par réaction, du Texte-branle - mais mieux : un Texte Tuteur, notion qui fait passer à une réalité (une esthétique) structurale : cf. Vita Nova ${ }^{25}$.

Concernant le "moi», les documents sont encore indisponibles ${ }^{26}$. On peut néanmoins s'en faire une idée, notamment grâce aux témoignages repris par les biographies de Barthes, comme, par exemple, l'ouvrage de Louis-Jean Calvet :

23. Fiche 68I, verso. (Tous les documents du Fonds Roland Barthes ont été transcrits par nous.)

24. Fiche 43I, verso.

25. Fiche 428 , recto.

26. La direction de l'IMEC soutient que la famille de Barthes s'oppose à la divulgation des textes concernant la vie privée des tiers. 
Jean-Louis Bouttes qui, à cette époque, parle longuement avec lui, pense que ces notes font partie d'un projet de roman. Il mettait en fiches des personnages rencontrés au hasard des soirées, brossait aussi aux amis des portraits croqués sur le vif, avec humour et précision, avec parfois aussi une méchanceté sèche ${ }^{27}$.

On peut considérer l'interdiction de consulter le fichier complet comme un indice de la présence d'un travail sur le «moi» de Barthes, travail très privé à première vue, et qui pourrait susciter des réserves analogues à celles soulevées par la publication du Journal de deuil. Ce Journal correspondait d'ailleurs à une partie du fichier et à une partie de Vita Nova; et c'est dans ce journal que j'ai trouvé peut-être l'indice le plus clair de l'existence des fiches dédiées à la transformation des personnes privées en personnages : "9 mars 1979. Maman et la pauvreté; sa lutte, ses déboires, son courage. Sorte d'épopée sans attitude héroïque ${ }^{28}$.»

\section{Du côté critique: réécrire Proust, c'est perdre son temps}

À côté de cette première voie, constituée par le projet de réécrire La Recherche, il faut considérer l'autre direction, la démystification, que l'on retrouve surtout dans les notes préparatoires de Vita Nova. Tout en affirmant sa volonté de le réécrire, Barthes met en question le roman proustien, et cherche des chemins d'écriture différents, voire contraires. Comme dans les Fragments (où Barthes ne présente jamais Werther comme un mythe de la petite bourgeoisie), la démythification ne se fait pas ici ouvertement : on ne peut en effet trouver des critiques explicites à la démarche proustienne.

Il faut donc chercher des indications dans La Préparation du roman et dans le Journal de deuil. Dans les premières séances du cours, par exemple, Barthes présente La Recherche comme un mythe qu'il essaierait de suivre : le mythe du vouloir-écrire, ou le scripturire. Pour décrire ce mythe, le professeur l'oppose aux autres mythes :

[...] tout récit mythique récite (met en récit) que la mort sert à quelque chose.

Pour Proust : écrire sert à sauver, à vaincre la Mort. Non pas la sienne, mais celle de ceux qu'on aime, en portant témoignage pour eux, en les perpétuant, en les érigeant hors de la non-Mémoire ${ }^{29}$.

Or, le moyen de Proust pour vaincre la mort - même si Barthes ne l'explicite pas - est bien connu : il suffit de relire l'extrait qui précède le célèbre passage du Temps retrouvé, où le narrateur proustien affirme que «la seule vie par conséquent réellement vécue, c'est la littérature». Dans cet extrait, il est

27. L.-J. Calvet, Roland Barthes (I9I5-I980), Flammarion, I990, p. 284.

28. R. Barthes, Journal de deuil, op. cit., p. 24I.

29. R. Barthes, La Préparation du roman, op. cit., p. 34. 
d'abord question de critiquer la "littérature de notations» (les journaux) qui prétend peindre le présent, la vie quotidienne :

Comment la littérature de notations aurait-elle une valeur quelconque, puisque c'est sous des petites choses comme celles qu'elle note que la réalité est contenue (la grandeur dans le bruit lointain de l'aéroplane, dans la ligne du clocher de Saint-Hilaire, le passé dans la saveur d'une madeleine, etc.) et qu'elles sont sans signification par elles-mêmes si on ne l'en dégage pas?

Peu à peu, conservée par la mémoire, c'est la chaîne de toutes ces expressions inexactes où ne reste rien de ce que nous avons réellement éprouvé, qui constitue pour nous notre pensée, notre vie, la réalité, et c'est ce mensonge-là que ne ferait que reproduire un art soi-disant "vécu ", simple comme la vie, sans beauté, double emploi si ennuyeux et si vain de ce que nos yeux voient et de ce que notre intelligence constate qu'on se demande où celui qui s'y livre trouve l'étincelle joyeuse et motrice, capable de le mettre en train et de le faire avancer sa besogne. La grandeur de l'art véritable, au contraire, de celui que M. de Norpois eût appelé un jeu dilettante, c'était de retrouver, de ressaisir, de nous faire connaître cette réalité loin de laquelle nous vivons, de laquelle nous nous écartons de plus en plus au fur et à mesure que prend plus d'épaisseur et d'imperméabilité la connaissance conventionnelle que nous lui substituons, cette réalité que nous risquerions fort de mourir sans avoir connue, et qui est simplement notre vie ${ }^{30}$.

Selon Proust, quand bien même aurait-on la plus superficielle des vies, le contact avec l'art véritable nous permet de retrouver le temps, qui a toujours été perdu. Il y a ainsi quelque chose de triste et d'heureux dans l'écriture : la vie que l'on a vécue ne signifie rien, mais elle peut, du coup, devenir une vie véritable. Nous pouvons passer notre vie dans la plus parfaite des aliénations et dans un manque complet d'engagement envers nous-mêmes et le monde. Mais un contact plus profond avec l'art nous permet d'avoir une autre vie, «la seule vie véritablement vécue». Du point de vue des Mythologies, ce petit récit relève d'un mythe bourgeois. L'art devient ainsi une façon de vaincre la mort, la mort de tous les jours, la mort des ennuis quotidiens, la mort contenue dans les toutes petites choses de la réalité, comme le bruit lointain de l'aéroplane, la ligne du clocher de Saint-Hilaire, la saveur d'une madeleine... La vie nouvelle de Barthes coïncide avec cette vie de l'écriture, où tout, êtres et choses disparus, reviendrait d'une façon véritable. On peut observer cette oscillation entre pensée mythique et démythification dès le premier chapitre du premier plan de Vita Nova: «I. Le monde comme objet contradictoire de spectacle et d'indifférence [comme discours $]^{3 \mathrm{H}}{ }^{\mathrm{n}}$. Le premier chapitre du livre porte exactement sur cette mort de la vie quotidienne dont parle le narrateur

30. M. Proust, Le temps retrouvé, dans À la recherche du temps perdu, Gallimard, coll. «Bibliothèque de La Pléiade», t. IV, I989, p. 473-474.

3I. R. Barthes, "Vita Nova», art. cité, p. I008. Les crochets et l'italique indiquent, dans la transcription, une insertion ultérieure. 
proustien. Toutefois, l'ajout au crayon ("[comme discours $]$ ») nous montre qu'à un autre moment de l'écriture, Barthes change d'avis et ne regarde plus ce premier chapitre comme une vérité, mais seulement comme un "discours" - et, j'oserais même dire, comme un mythe. Le Journal de deuil correspond pleinement à cette prise de conscience face au mythe. Maintes fois dans ses notes, Barthes renvoie au besoin d'écrire pour ne pas oublier (ou pour faire vivre ce qui est mort); mais, en avril 1978, quelque chose change dans son désir :

Casa, 2I avril 1978.

Pensée de la mort de mam. : brusques et fugitives vacillations, fadings très courts, prises poignantes et cependant comme vides, dont l'essence est : la certitude du Définitif ${ }^{32}$.

À partir de ce moment du Journal, Barthes devient conscient que l'écriture, comme il l'avait déjà mentionné dans les Fragments d'un discours amoureux, "ne compense rien, ne sublime rien, qu' elle est précisément là où tu n'es pas $^{33}$ ». La démythification est son second deuil, le deuil de la mort définitive, qui le poussera à chercher d'autres possibilités d'écriture.

\section{Pour ne pas perdre son temps}

\section{Noter le présent}

Au tout début du cours, lors de la séance du I6 décembre 1978, Barthes se pose une question qui détruira son projet mythique initial :

Dans l'état actuel de ma réflexion, j'ai donc le sentiment que le Roman fantasmé ne peut être, en ce qui me concerne, de type anamnésique. La "pulsion" romanesque (amour du matériau) ne va pas vers mon passé. Ce n’est pas que je n'aime pas mon passé, c'est plutôt que je n’aime pas le passé (peut-être parce qu'il déchire), et ma résistance prend la forme de cette brume, dont j'ai parlé $\rightarrow$ sorte de résistance générale à réciter, à raconter ce qui ne reviendra plus (le rêve, la drague, la vie passée). Le lien affectif est avec le présent, mon présent, dans les dimensions affectives, relationnelles, intellectuelles = le matériau que je souhaite ${ }^{34}$

Or, il est impossible de lier une récriture de La Recherche à un refus du passé; bien plus, Barthes ne va pas seulement abolir le passé, il va aussi utiliser le genre d'écriture auquel s'oppose Proust : la notation de la vie quotidienne.

32. R. Barthes, Journal de deuil, op. cit., p. I24.

33. R. Barthes, Fragments d'un discours amoureux, op. cit., p. I32.

34. R. Barthes, La Préparation du roman, op. cit., p. 45. 
Il faut ici mentionner la pluralité discursive de Vita Nova:

IV La litt. comme déception (c'était une Initiation)

Le déjà fait : l'Essai

Le Fragment

Le Journal

Le Roman

[- Le Comique?]

La Nostalgie ${ }^{35}$.

Dès les premiers plans, il est ainsi prévu que le livre contienne d'autres textes, et comme on le lit dans la chemise Vita Nova, la question principale de l'écriture devient : "comment organiser ces textes différents" (folios 6 et $7)^{36}$. Voici un exemple de cette préoccupation :

[Lisant Pascal] Envie de :

$<$ Frag $>$ Faire comme si je devais écrire ma grande ouvre (Somme) <la illis. $>-$ mais Apologie de quoi? là est la question! En tout cas pas de «moi»!) - et qu'il n'en restât que des ruines ou linéaments, ou parties erratiques (comme le pied peint par Porbus) : des Fragments d'inégale longueur <peut-être des illis.> (ni aphorismes ni dissertations $)^{37}$.

Ces folios portent sur un projet apparemment nouveau, inspiré de Pascal et composé des fragments d'une apologie de "quelque chose», qui n'est pas encore défini. Dans le fonds Barthes, à l'IMEC, j’ai trouvé quatre folios qui peuvent passer pour la suite de ce développement initial, classé dans la chemise Vita Nova. D'après ce qu'on peut lire dans ces inédits, Barthes voulait récrire les Pensées, une œuvre fragmentaire présentant plusieurs ordres possibles - mais qui produirait chez le lecteur l'effet d'un grand «Bien» commun.

Ce projet ne nie pas celui du roman de Vita Nova. L'apologie sert apparemment de modèle pour intégrer différents textes déjà écrits, surtout des textes de notations, des journaux :

On aurait :

INCIDENTS (amalgame avec APOLOGIE - et neutre)

Precédé? Introduit par Récit : Deuil, Vita Nova, Bilan, Examen de conscience, Table Rase etc ${ }^{38}$.

Incidents, Vita Nova et le Journal de Deuil, et probablement «Soirées de Paris» (des récits qui racontent la vie quotidienne de Barthes, et notamment cette vie «secrète» qu'il cachait soigneusement), correspondent à des «liasses »,

35. R. Barthes, "Vita Nova», art. cité, p. Ioo8.

36. Ibid., p. IOI5-IoI7.

37. Ibid., p. IOI6.

38. Il s'agit du deuxième feuillet du manuscrit titré «Incidents» et conservé à l'IMEC. 
des «sujets» de fragments, comme les sous-titres des Pensées. Comme chez Pascal, l'apologie barthésienne devait partir du mondain, du «divertissement» et, par là, entrer (et faire entrer le lecteur) dans la sphère du sacré.

Le sacré

Il nous reste à comprendre ce que ce sacré pouvait signifier pour Barthes. Dans les manuscrits publiés, la question est posée, comme on l'a vu au feuillet 7 ("mais Apologie de quoi?/là est la question! En tout cas pas de moi!»). Dans les manuscrits que nous avons pu consulter, la définition de ce sacré se précise :

Idée (qui s'empare de moi de + en + à chaque fiche) d'un auto-commentaire (lorsq aps-coup) $\rightarrow$ menant le travail vers le Bien, pour devenir «bien», parfait, moral (vers la mère). Initiation. Religion

$\mathrm{cf}=$ confessions d'un écrivain ${ }^{39}$.

Pour Barthes, il n'était pas question de Dieu ou de la religion : son sacré était de l'ordre de l'amour, non un amour platonique comme celui des Fragments, mais l'amour pour un "objet» avec lequel on peut - et il a $\mathrm{pu}$ - vivre ensemble (Barthes a vécu avec sa mère toute sa vie). De même, l'utopie de son premier cours consiste à faire vivre ensemble ses liasses, ses récits incomplets, ses projets inachevables.

Ainsi, Barthes revient, malgré tout, à l'idée de faire vivre ceux qu'il aime, surtout celle dont la mort lui a causé tant de chagrin : la mère. Mais la mère n'est plus un personnage, elle n'est plus quelqu'un appartenant au passé : elle est devenue une façon de se regarder, de rassembler ses écrits. Vita Nova pourrait dès lors figurer comme un retour aux Mythologies : par ce projet, Barthes pose à la fois l'écriture comme mythe, et l'amour (la mère) comme démythification. Bien sûr, ce ne sera pas la mère de Barthes, figure de l'amour et de la générosité, qui regardera ses écrits, mais chaque lecteur, comme opérateur de ce processus de démythification, prendra sa place et la fera revivre à chaque lecture :

La plupart de mes observations sont discutables récusables - au bénéfice précisément d'une plus grande intelligence, générosité.

Concevoir un discours à 2 volets : la note et son dépassement, d'où surgit la valeur véritable auto-critique, mais comme dépassement et découverte non de la valeur mais d'une manière de la poser ${ }^{40}$.

39. Se reporter toujours au deuxième feuillet du manuscrit «Incidents". 40. Voir le premier feuillet du manuscrit «Incidents». 